\title{
10.2478/acsc-2014-0026
}

\section{Intelligent control algorithm for ship dynamic positioning}

\author{
WANG MENG, LI HONG SHENG, MIAO QING and BIAN GUANG RONG
}

\begin{abstract}
Ship motion in the sea is a complex nonlinear kinematics. The hydrodynamic coefficients of ship model are very difficult to accurately determine. Establishing accurate mathematical model of ship motion is difficult because of changing random factors in the marine environment. Aiming at seeking a method of control to realize ship positioning, intelligent control algorithms are adopt utilizing operator's experience. Fuzzy controller and the neural network controller are respectively designed. Through simulations and experiments, intelligent control algorithm can deal with the complex nonlinear motion, and has good robustness. The ship dynamic positioning system with neural network control has high positioning accuracy and performance.
\end{abstract}

Key words: dynamic positioning, fuzzy control, neural network control, BP algorithm

\section{Introduction}

According to the International Maritime Organization and other certifying class societies, a dynamically positioned vessel is defined as a vessel that accurately maintains its position and heading at the fixed location or pre-determined track for marine operation purposes exclusively by means of active thrusters [1]. Many marine working ships have been equipped with dynamic positioning system (DPS) to maintain stable working conditions. DPS is generally consisting of position measurement system, control system and thruster system. As ocean development moves to deeper and farther, DP technology has more and more important practical significance and has received more and more attention [2]. DPS is a very complex system. The motion of a ship is affected by uncertain environmental forces with a broad frequency spectrum, which imposes the necessity of including complex control and filtering algorithms into the DPS. Furthermore, the dynamics of a ship is modeled by a highly nonlinear equation that makes the design of such algorithms an even harder task [3].

DPS has been applied on vessel since the 1960s, and today it is equipped on many new vessels used for freight transport, offshore exploration and exploitation [4]. So far many control techniques are used in DPS [5-9]. In the 1960s PID controller began to

The Authors are with Wuhan University of Technology, Mechanic and Electronic Engineering, Wuhan, China. Corresponding Author is Wang Meng, email: 731768832@qq.com.

Received 12.09.2014. Revised 05.11.2014. 
apply in DPS to control the motion of three directions (surge, sway and yaw). This product is the first-generation DPS which utilized conventional PID control technology and low-pass filter technology. Subsequently, control technology based on the modern control theory is applied to DPS. That is known as the second-generation product of DPS. Since then, Nonlinear PID-control, passive observer design and observer backstepping design have been applied [7, 10-12]. Under the promotion of Balchen, Jenssen, Saelid and other scholars, control technology in DPS is further developed. Since the end of last Century, the third-generation DPS based on intelligent control theory and method begin to attract considerable attention, such as robust control, fuzzy control [13-15], nonlinear model predictive control, neural network control [16] etc. In this generation, DPS control technology is making progress on the trend of intelligent. Fuzzy logic systems (FLS) and neural networks (NN) have been successfully applied to many control problems because they do not need an accurate mathematical model of the system. It is also known that these intelligent systems can approximate uniformly any nonlinear continuous function over a compact set $[2,3]$.

Ship motion is a big lag time and complex nonlinear kinematics. The hydrodynamic coefficients of ship model are very difficult to accurately determine, and dynamically vary with the change of environment. In recent years, intelligent control approaches have been put forward to deal with dynamic uncertainties of nonlinear systems via FLS [17] or NN [18]. This paper considers uncertainties of both dynamic and disturbance in DPS control design, which is more practical. By summarizing operator's experience to take the corresponding decision, two kind of robust nonlinear controller are proposed to realize positioning.

This paper takes a $3.5 \mathrm{~m}$ test ship as the research object. The main objective is to use intelligent control law to keep the position and heading of the vessel at a set value, and be able to guarantee high control precision under certain marine environments. In addition, the control law should be satisfied the thrust allocation algorithm and propulsion system physical limitation. Decoupling control theory combining with FLS theory and neural network control theory are mainly used in this paper. Intelligent controllers are designed for 3-DOF. Simulations and experiments are conducted, taking into account of the facts such as complicated marine environment conditions, uncertainty of vessel model, and physical limits of ship thruster system. The simulation results are analyzed and studied.

\section{Mathematical model and control system}

\subsection{Mathematical model}

Motion modeling of ocean vessel involves the study of kinematics and dynamics. Kinematics is concerned with the change of position, velocity, acceleration, attitude and angular velocity versus time. Dynamics is concerned with how the ship changes position and attitude by force and torque. Under the complicated marine conditions, the vessel will produce six direction's movement, namely, sway, surge, yaw, roll, pitch and heave 
as shown in Fig. 1. In DPS, it has been adequate to regard the control objective as the 3 degree of freedom (3-DOF) problem in the horizontal plane in surge, sway and yaw respectively.

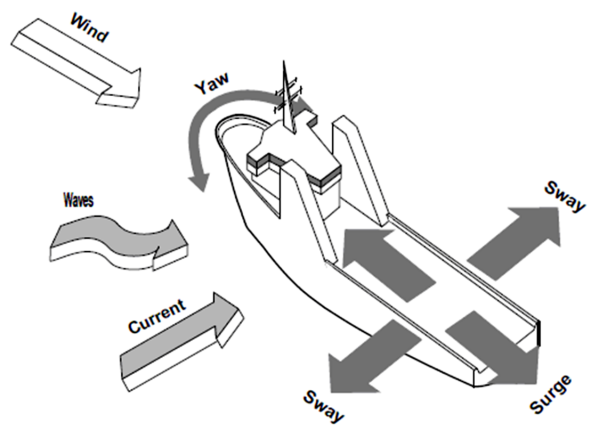

Figure 1: Ship motion.

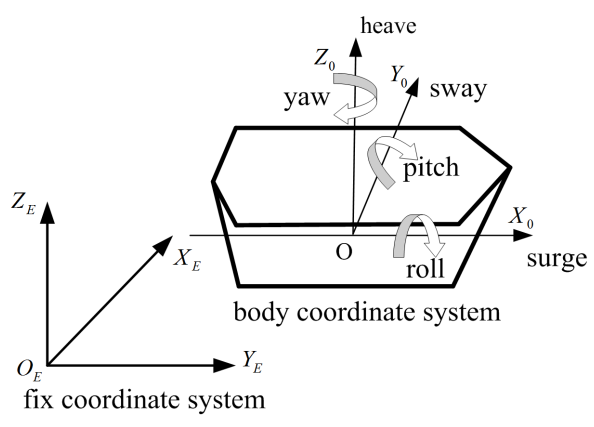

Figure 2: Two kinds of coordinate reference system.

For the analyzes of the motion, it is convenient to define two coordinate frames, fixed coordinate frame and body coordinate frame [19], as indicated in the Fig. 2.

$O-X_{0} Y_{0} Z_{0}$ is the body coordinate system, which is fixed to the vessel, and the origin is usually chosen to be the center of gravity of the vessel. $O_{E}-X_{E} Y_{E} Z_{E}$ is the fixed coordinate system, which is fixed at a point of the earth's surface. Based on the Euler's theorem on rotation, the kinematic equations relating the body coordinate frame to the fixed coordinate frame can be expressed in vector for as $\dot{\eta}=R(\psi) v$ where $R(\psi)$ is the transformation matrix. Its expression is

$$
R(\psi)=\left[\begin{array}{ccc}
\cos (\psi) & -\sin (\psi) & 0 \\
\sin (\psi) & \cos (\psi) & 0 \\
0 & 0 & 1
\end{array}\right]
$$


$\eta=\left[\begin{array}{lll}x & y & \psi\end{array}\right]^{T}$ represents the position and heading in the fixed coordinate system.

$v=\left[\begin{array}{lll}u & v & r\end{array}\right]^{T}$ represents surge velocity, sway velocity and rolling angular velocity in the body coordinate system.

According to the basic theorem of kinematics and dynamics, there are certain relationship equations between the force and the movement. This relationship equation can be expressed in a system of equations [20]:

$$
\left\{\begin{array}{c}
\dot{\eta}=R(\psi) v \\
M \dot{v}+D v=\tau
\end{array}\right.
$$

where $M$ is the added mass due to the inertia of the surrounding fluid and $D$ is the hydrodynamic damping matrix. There structure is as follows:

$$
M=\left[\begin{array}{ccc}
m_{11} & 0 & 0 \\
0 & m_{22} & m_{23} \\
0 & m_{32} & m_{33}
\end{array}\right], \quad D=\left[\begin{array}{ccc}
d_{11} & 0 & 0 \\
0 & d_{22} & d_{23} \\
0 & d_{32} & d_{33}
\end{array}\right]
$$

$\tau=\left[\begin{array}{lll}X & Y & N\end{array}\right]$ is the force and moment in the ship coordinate system.

In this paper, the control object is the test ship. By the experimental method, inertia matrix and damping matrix is obtained. They are as follows:

$$
M=\left[\begin{array}{ccc}
0.754 & 0 & 0 \\
0 & 1.199 & 0.211 \\
0 & 0.029 & 0.524
\end{array}\right], \quad D=\left[\begin{array}{ccc}
0.014 & 0 & 0 \\
0 & 0.102 & -0.024 \\
0 & 0.192 & 0.095
\end{array}\right]
$$

\subsection{Control system}

According to the requirement of DPS, we should keep the position and heading at set value, or make them move to the set point and the heading. The actual ship motion model and precise mathematical model of outside environment interference is difficult to describe. Artificial intelligence techniques methods, fuzzy set theory and neural network expert system, used in the DP control system, overcome the uncertainty and nonlinearity of model. This paper designed intelligent controller and feed-forward decoupling compensator respectively. The block diagram of control system is shown as Fig. 3 .

In the structure, position $\left(x_{s e t}, y_{\text {set }}\right)$ and heading $\psi_{\text {set }}$ are set in fix coordinate system through console. Three intelligent controllers are corresponding designed for three directions. In fix coordinate system, each controller calculates force $\left(F_{X}, F_{Y}\right)$ and torque $N$ according to the error and change of error. The error signal is calculated by the difference between set value and actual value from sensors. The output of controllers will be transformed to force and torque in body coordinate system through transformation matrix $R^{-1}(\psi)$. Decoupling control is designed to remove the decoupling between sway loop and yaw loop. The force from wind, wave and flow, as disturbance, acts to the vessel. 


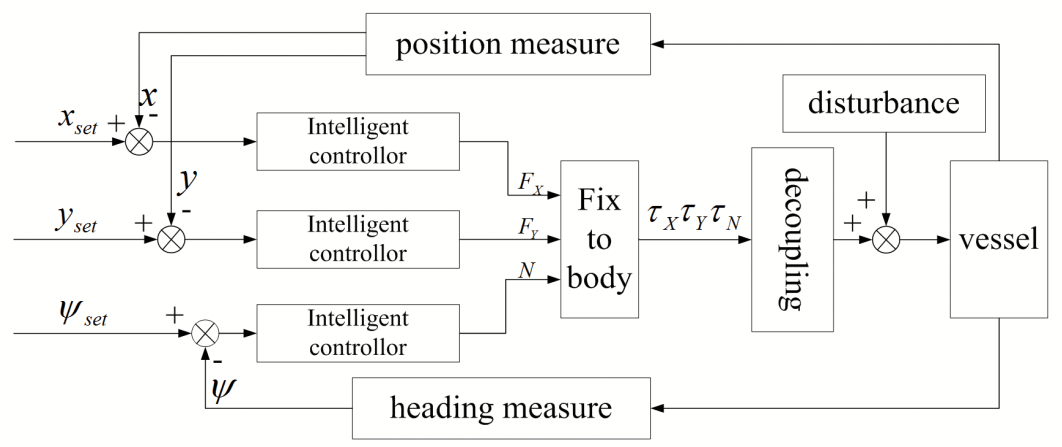

Figure 3: Block diagram of control system.

\section{Fuzzy control for DPS}

As an intelligent method, fuzzy logic controller (FLC) is widely used in industrial processes due to its inherent robustness [21]. Fuzzy control strategy is proposed for DPS in this paper. We consider a two inputs and one output fuzzy system. The prime objective of the controller design is to achieve a good control performance in terms of the stability and the robustness for the system uncertainties and the environmental disturbances.

\subsection{Structure of the fuzzy logic system}

The basic configuration of a fuzzy logic system consists of a fuzzifier, fuzzy IFTHEN rules, a fuzzy inference engine and a defuzzifier as shown in Fig. 4. Fuzzifier, which is the fuzzy quantizer of the inputs, allows the conversion of the inputs variables which are physical quantities, in fuzzy quantities, or linguistic variables. Defuzzifier is the inverse operation of the fuzzifier. It consists in transforming the linguistic variables into real or digital variables [22].

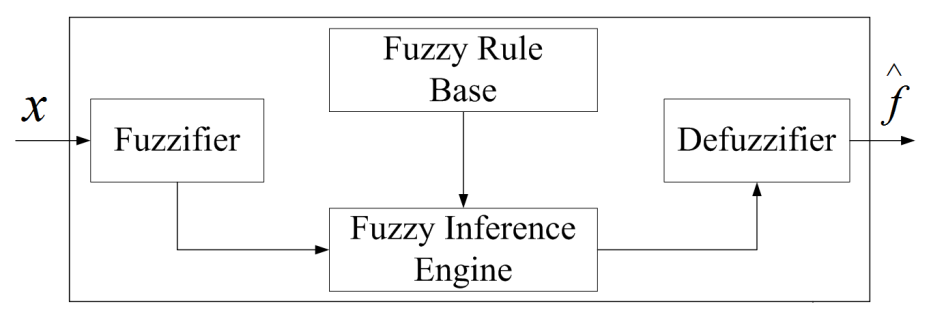

Figure 4: Fuzzy logic system.

The fuzzy inference engine uses the fuzzy IF-THEN rules to perform a mapping from an input vector $x^{T}=\left[x_{1}, x_{2}, \cdots, x_{n}\right] \in \mathfrak{R}^{n}$ to an output $\hat{f} \in \mathfrak{R}$. The $i$ th fuzzy rule is written as $R^{(i)}$ : If $x_{1}$ is $A_{1}^{i}$ and $\ldots$ and $x_{n}$ is $A_{n}^{i}$ then $\hat{f}$ is $f^{i}$ where $A_{1}^{i}, A_{2}^{i}, \ldots$ and $A_{n}^{i}$ are 
fuzzy sets and $f^{i}$ is the fuzzy singleton for output in the $i$ th rule. By using the singleton product inference, and center-average defuzzifier, the output of the fuzzy system can be expressed as follows:

$$
\hat{f}(x)=\frac{\sum_{i=1}^{m} f^{i}\left(\Pi_{j=1}^{n} \mu_{A_{j}^{i}}\left(x_{j}\right)\right)}{\sum_{i=1}^{m}\left(\Pi_{j=1}^{n} \mu_{A_{j}^{i}}\left(x_{j}\right)\right)}=\theta^{T} \psi(x)
$$

where $\mu_{A_{j}^{i}}\left(x_{j}\right)$ is the degree of membership of $x_{j}$ to $A_{j}^{i}, m$ is the number of fuzzy rules, $\theta^{T}=\left[f^{1}, f^{2}, \cdots, f^{m}\right]$ is the adjustable parameter vector (composed of consequent parameters), and $\psi^{T}=\left[\psi^{1}, \psi^{2}, \cdots, \psi^{m}\right]$ with

$$
\psi^{i}(x)=\frac{\Pi_{j=1}^{n} \mu_{A_{j}^{i}}\left(x_{j}\right)}{\sum_{i=1}^{m}\left(\Pi_{j=1}^{n} \mu_{A_{j}^{i}}\left(x_{j}\right)\right)}
$$

being the fuzzy basis function[17].

\subsection{Design of the FLC for DPS}

In this paper, three FLCs are design for 3-DOF. Take surge direction as example. The input values are $e$ and $e c$ in accordance with error signal of $x$ direction and its variation. The output value is $u$ in accordance with controlled value. With regard to ship motion, the parameter domain for the surge loop is as follows:

$$
\left\{\begin{array}{l}
e \in[-5,5] \\
e c \in[-5,5] \\
u \in[0.03,0.03]
\end{array}\right.
$$

The linguistic terms of each value ( $e, e c$ and $u$ ) is divided into seven fuzzy sets. These are characterized by the following standard designations: negative big $(N B)$, negative middle $(N M)$, negative small $(N S)$, zero $(Z)$, positive small $(P S)$, positive middle $(P M)$, and positive big $(P B)$. For convenience, membership functions (MFs) of inputs and output are chosen as triangle-shaped MF. Fig. 5 shows the MFs for the error signal, change of error signal, and output respectively.

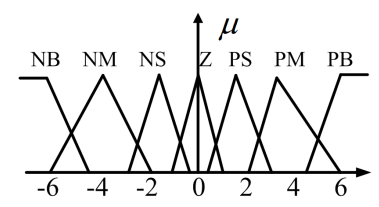

(a)

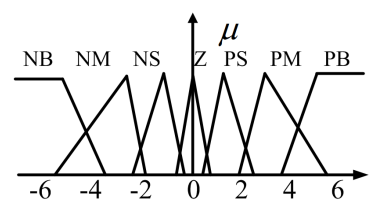

(b)

Figure 5: MFs of inputs (a) and output (b).

The basis of the rules represents the strategy of control and the desired aim is determined by the linguistic control rules [23]. Considering the characteristics of ship motion, 
the control rules are established as Tab. 1. After the fuzzy output is calculated, it will be transformed into a numeric value which can be recognized by thruster allocation algorism in DPS. In this work, the method of center-average defuzzification previously introduced is applied.

Table 5: Control rules.

\begin{tabular}{cccccccc}
\hline & \multicolumn{8}{c}{$\boldsymbol{D U}$} \\
\cline { 2 - 7 } $\boldsymbol{E} C$ & \multicolumn{7}{c}{$\boldsymbol{E}$} \\
\cline { 2 - 8 } & NB & NM & NS & $\boldsymbol{Z}$ & PS & PM & PB \\
\hline NB & NB & NB & NB & NM & NS & PS & PM \\
\hline NM & NB & NB & NM & NM & Z & PS & PM \\
\hline NS & NB & NB & NS & NS & Z & PM & PM \\
\hline $\boldsymbol{Z}$ & NB & NM & NS & Z & PS & PM & PB \\
\hline PS & NM & NM & Z & PS & PS & PB & PB \\
\hline PM & NM & NS & Z & PM & PM & PB & PB \\
\hline PB & NM & NS & PS & PM & PB & PB & PB \\
\hline
\end{tabular}

\section{NN control for DPS}

In the fuzzy control, the performance is determined by fuzzy rules which are conducted by experience. This kind of operator's experience can also be used in NN control through utilizing the intelligent control rules to train the network, so as to design the $\mathrm{NN}$ controller. After finishing training the off-line network, the control quantity can be obtained through the online calculation. This kind of NN controller costs less computing time, and need not to establish the complex rule table. As neural network has the self-learning and associative functions, the output of network can also make a decision through the associative memory for new inputs excluded in the training samples.

In this paper, BP neural network is adopted to design the DPS controller. BP neural network is multi-layer perceptron network which uses the back propagation method to update the network weights. The multi-tier architecture description is to increase the network's description capacity for nonlinear problem. The learning process of the BP algorithm is composed of forward and backward propagation. In the forward propagation, input sample enters the network through the input layer, and then will participate in calculating process from hidden units to output units. During the processing, neurons' state of each layer only influences the neurons' state of next layer. In the output layer, 


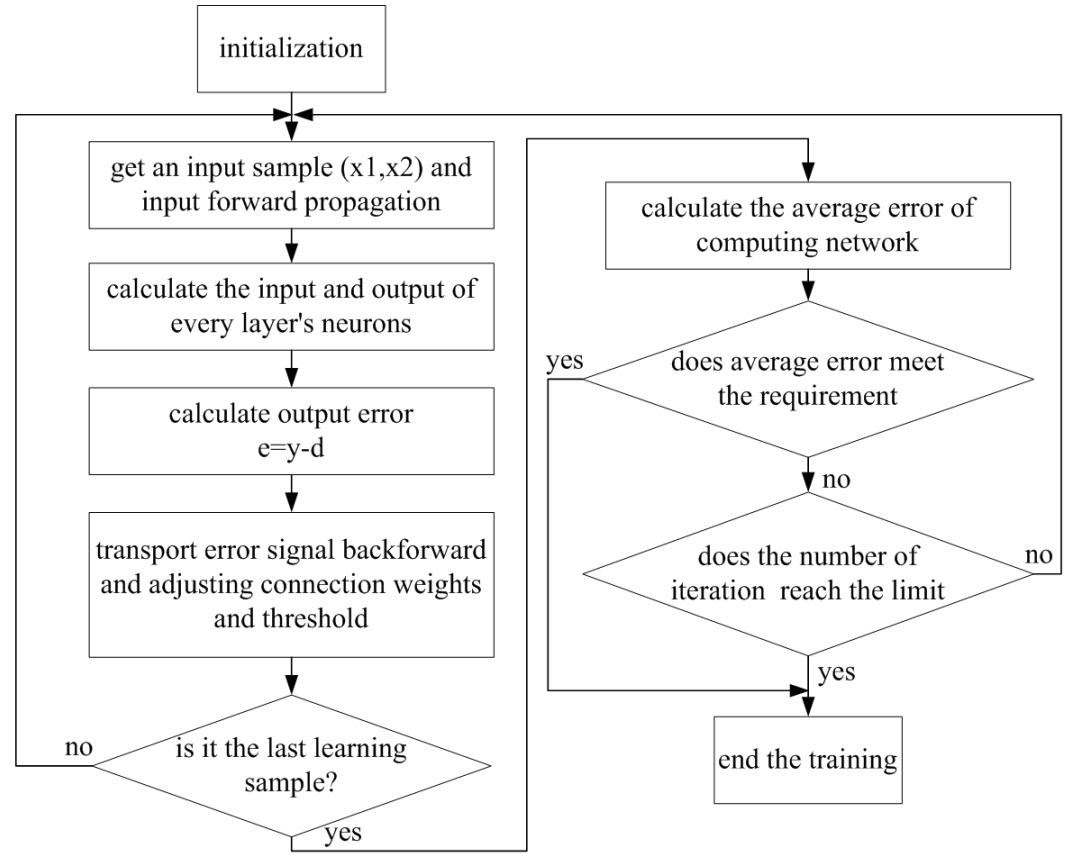

Figure 6: Process of BP algorithm.

current output and expected output are compared. If they are not equal, the process will get into the back propagation procedure.

The general process of $\mathrm{BP}$ algorithm is shown as Fig. 6. The number of iterations is given in order to end the operation program when the average error cannot meet the requirement of precision.

The architecture of NN controller for DPS is shown in Fig. 7. The control rules are conduct by a neural network, and the modification of control rules is finished by learning algorithm. The normalized inputs are calculated through multi-layer's neurons, and the output of network can be used as real control quantity after conversion.

In this paper, neural network is realized by using a 3 layers neural network. The structure is shown in Fig. 8. Vector $p=\left[x_{1}, x_{2}\right]$ is input sample of neural network. $w_{1}$ is weighting matrix of the hidden layer. $b_{1}$ is the offset vector of hidden layer. $w_{2}$ is weights matrix of the output layer. $b_{2}$ is the offset vector of output layer. $f$ and $g$ are transfer function of hidden layer and output layer respectively.

From Fig. 3, jth nodes' input of hidden layer can be described as $N e t_{j}=\sum_{i=1}^{2} x_{i} w_{i j}$. The output of $j$ th node is $O_{j}=f\left(\sum_{i=1}^{2} x_{i} w_{i j}\right)$ where $f$ is the transport function. The input of output layer is $N e t=\sum_{j=1}^{P} O_{j} w_{j}$ and output of network is $y=g(\mathrm{Net})$. 


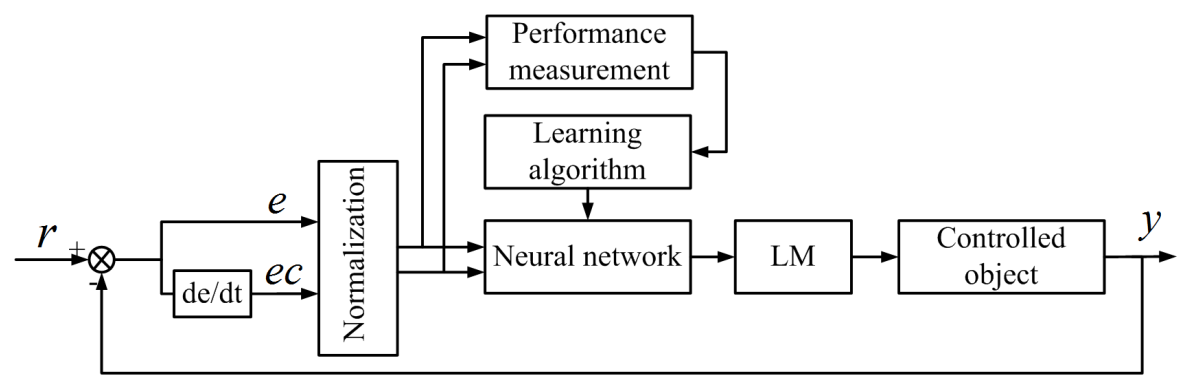

Figure 7: Architecture of NN control for DPS.

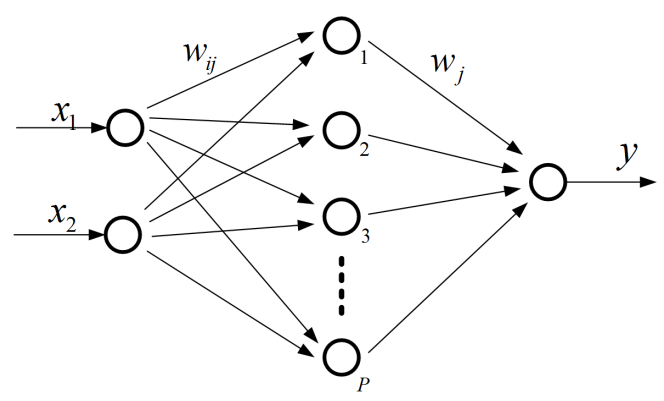

Figure 8: Three layers neural network.

In the forward propagation, the error signal will transport reverse to the original forward propagation path, and the weight coefficient of each individual neurons in hidden layer and output layer are modified, in order to make error signal tend to minimum.

Error function is defined as

$$
E=\frac{1}{2}(y-d)^{2}
$$

where $y$ and $d$ represent the real output and expected output. The BP algorithm modifies the weight coefficient according to the negative gradient direction of error function.

First, the weight coefficient of output layer is updated as

$$
w_{j}(k+1)=w_{j}(k)+\Delta w_{j}(k)=w_{j}(k)-\eta \frac{\partial E}{\partial w_{j}}
$$

where $\eta$ is the learning rate. The formula can be computed as:

$$
\Delta w_{j}=-\eta \frac{\partial E}{\partial w_{j}}=-\eta \frac{\partial E}{\partial y} \frac{\partial y}{\partial N e t} \frac{\partial N e t}{\partial w_{j}}
$$

where $\frac{\partial E}{\partial y}=y-d, \frac{\partial y}{\partial N e t}=g^{\prime}(N e t), \frac{\partial N e t}{\partial w_{j}}=O_{j}$. So the weight coefficient of output layer is updated as

$$
w_{j}(k+1)=w(k)-\eta g^{\prime}(N e t) O_{j}(y-d)
$$


Next, the weight coefficient of hidden layer is updated as

$$
w_{i j}(k+1)=w_{i j}(k)+\Delta w_{i j}(k)=w_{i j}(k)-\eta \frac{\partial E}{\partial w_{i j}}
$$

The formula can be computed as

$$
\Delta w_{i j}=-\eta \frac{\partial E}{\partial w_{i j}}=-\eta \frac{\partial E}{\partial O_{j}} \frac{\partial O_{j}}{\partial w_{i j}}=-\eta \frac{\partial E}{\partial y} \frac{\partial y}{\partial N e t} \frac{\partial N e t}{\partial O_{j}} \frac{\partial O_{j}}{\partial w_{i j}}
$$

where $\frac{\partial E}{\partial y}=y-d, \frac{\partial y}{\partial N e t}=g^{\prime}(N e t), \frac{\partial N e t}{\partial O_{j}}=w_{j}, \frac{\partial O_{j}}{\partial w_{i j}}=f^{\prime}\left(N e t_{j}\right) x_{i}$. So the weight coefficient of output layer is updated as

$$
w_{m}(k+1)=w_{m}(k)-\eta(y-d) g^{\prime}(N e t) w_{j} f^{\prime}\left(N e t_{j}\right) x_{i}
$$

49 fuzzy inference rules are used to train neural network. The ample set $[P, T]=$ $\left[X_{1}, X_{2}, Y\right]$ is extract from the rules. $X_{1} \in[-1,1]$ and $X_{2} \in[-1,1]$ represent the normalized input sample values of error and its variation. $Y$ represents the normalized expected value of output control force. After the training, control rules will be implied distribution in the whole neural network. The training results are as follows:

$$
\begin{aligned}
& w_{1}=\left[\begin{array}{llllllllll}
-3.08 & 0.656 & 0.565 & 0.410 & 0.548 & 0.618 & -3.04 & 0.546 & -3.65 & 0.577 \\
-2.98 & 0.519 & 0.513 & 0.453 & 0.450 & 0.520 & -2.96 & 0.496 & -3.18 & 0.501
\end{array}\right]
\end{aligned}
$$

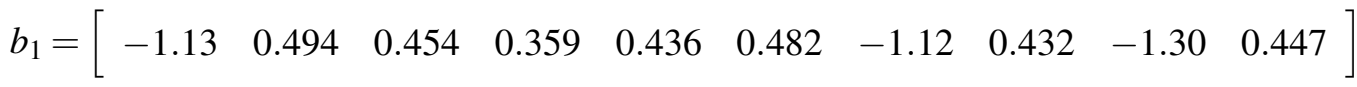

$$
\begin{aligned}
& w_{1}=\left[\begin{array}{llllllllll}
-0.0631 & 0.701 & 0.286 & 0.490 & 1.06 & 0.426 & 0.232 & 0.907 & 0.217 & 0.364
\end{array}\right] \\
& b_{2}=[-1.48]
\end{aligned}
$$

\section{Simulation and experiments}

In this section, simulations and experiments are carried out to demonstrate the effectiveness of the proposed intelligent control law. The model of test vessel is given as (10). The force from wind, wave and flow, as disturbance, acts to the vessel.

\subsection{Calm water case}

In calm water, the external load is zero. The initial states are chosen as $P_{0}=$ $[0 \mathrm{~m}, 0 \mathrm{~m}, 0 \mathrm{rad}]$. The desired position and heading are chosen as $y_{d}=[5 \mathrm{~m}, 5 \mathrm{~m}, 1 \mathrm{rad}]$. Figs. 9-12 show the simulation results in calm water. According to Figs. 9-10, displacement of position $(x, y)$ and displacement of heading $\psi$ process smoothly, and they have small 

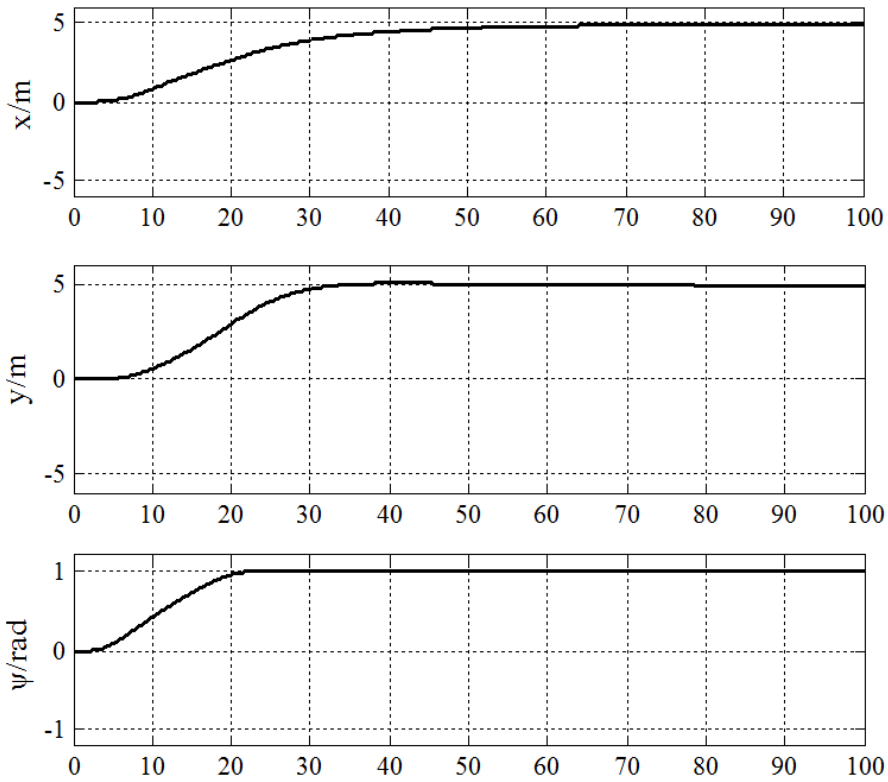

Figure 9: Displacement with NN control.
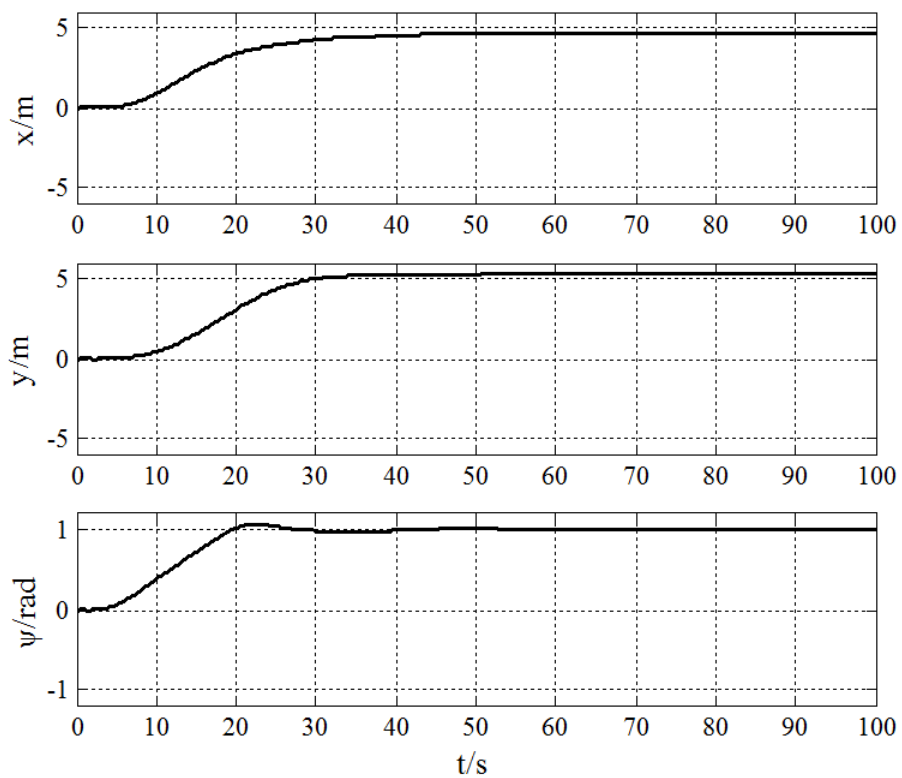

Figure 10: Displacement with fuzzy control. 

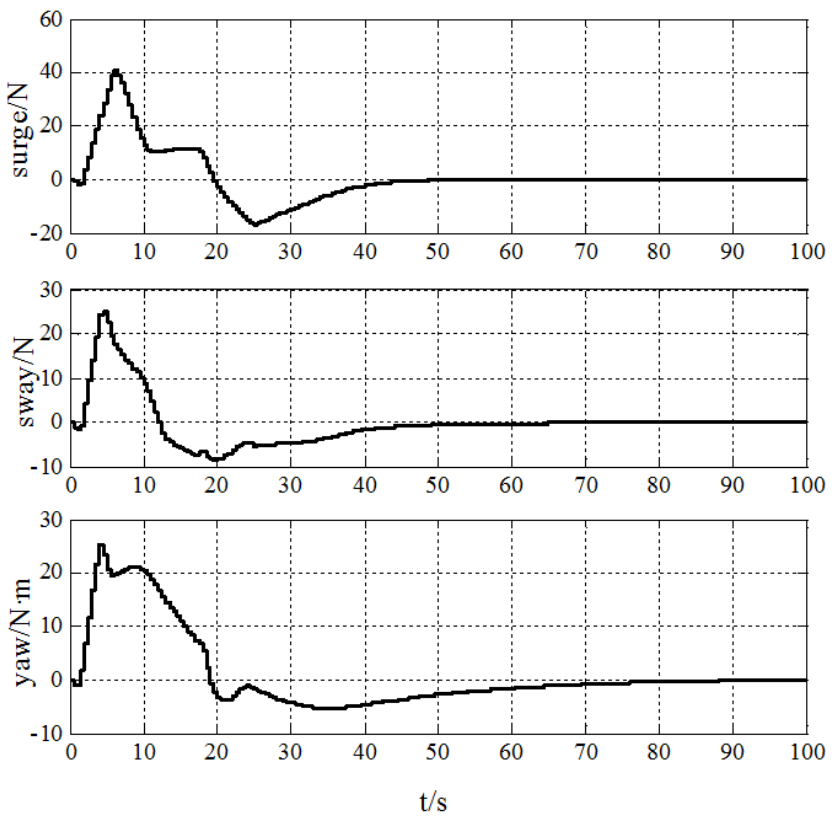

Figure 11: Output of NN control.
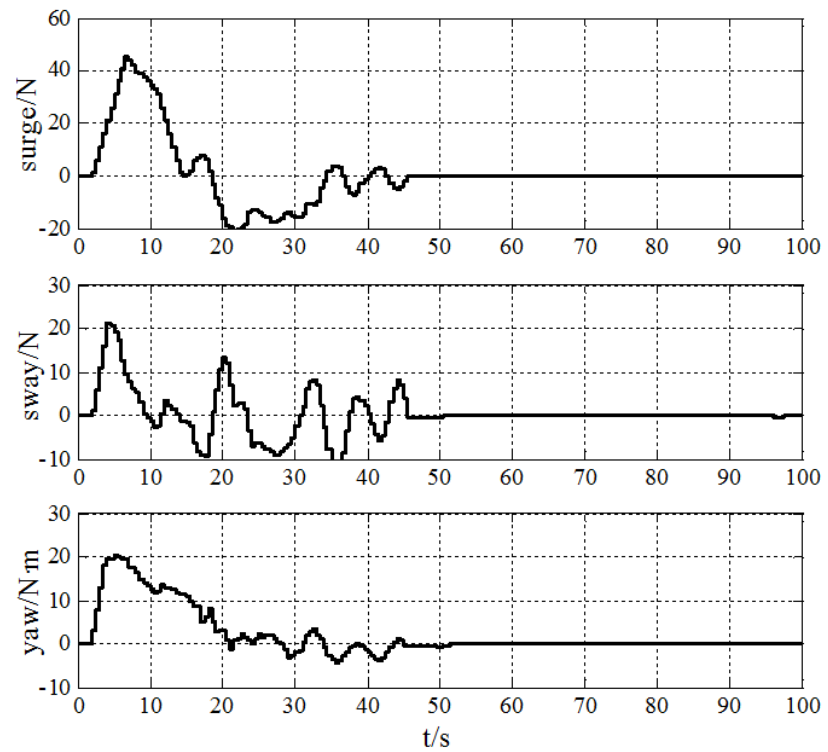

Figure 12: Output of fuzzy control.

overshoot. The actual ship position and the actual heading can approach to the desired position and heading $P_{d}=[5 \mathrm{~m}, 5 \mathrm{~m}, 1 \mathrm{rad}]$ in approximately $40 \mathrm{~s}$. 

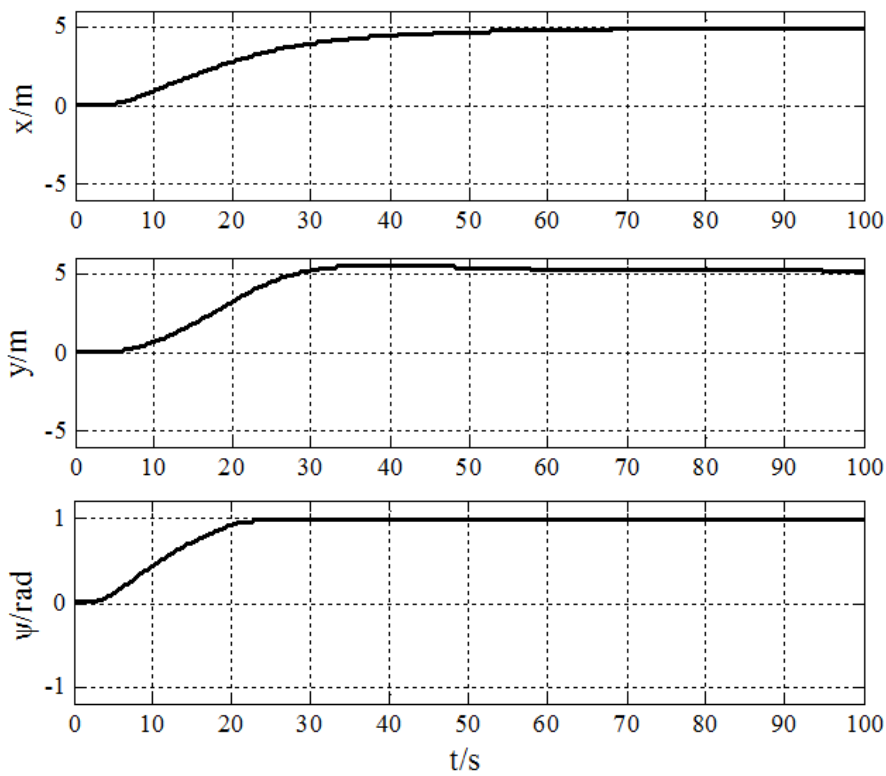

Figure 13: Displacement under disturbance with NN control.
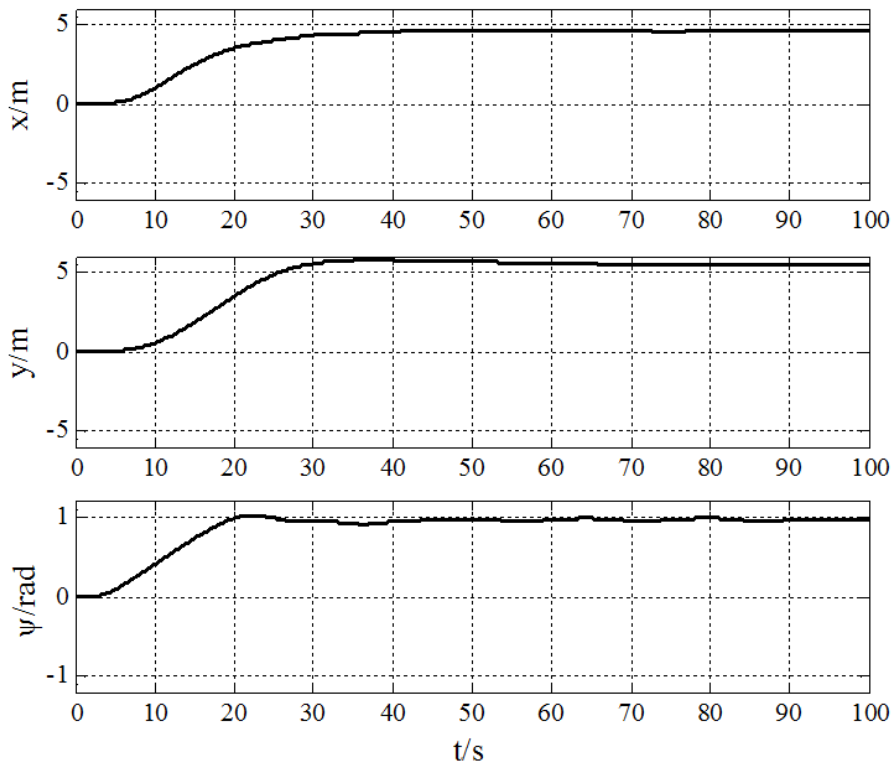

Figure 14: Displacement under disturbance with fuzzy control. 
Comparing Fig. 11 with Fig. 12, neural network control has better performance in output of force and torque compared to fuzzy control. At the beginning of control process, outputs of neural controller gradually achieve a larger value in order to force ship move fast. Along with approaching to the desire position and direction, the output will return to a smaller value or zero. Smoothly changing of control force and control torque of NN controller is easy to physical implementation, while outputs of fuzzy controller vibrate severely during the adjustment period, which will consume more energy.

\subsection{Disturbances}

In the simulations, disturbance is considered. In order to simulate external disturbance, force disturbance and torque disturbance are added. The disturbance is within range of $1 N(N \cdot m)-5 N(N \cdot m)$ considering the actual situation of test vessel. Figs. 1314 are the simulation result in external disturbance condition. By both NN control and fuzzy control, the vessel can still reach position, and continue to resist to the external load. The residual error of NN control is less than that of fuzzy control.

\subsection{Changed model}

As parameters of ship motion model are based on the water experiments, vessel's accurate model is difficult to be determined. By adding object properties disturbance module, we simulate a changed controlled object. So the transfer function of the object will be dynamically changed. The simulation results are depicted in Figs. 15-16. The results show that the proposed intelligent control is able to force the ship to converge to the desired position. Although the character of the controlled object (transfer function) is changed, the response curves of the controlled variable could slightly change. The result shows that intelligent control method has good robustness.

Through the simulation results and comparison above, intelligent control satisfies the positioning requirement. It has the ability to resist external interference, and still can make the ship to reach the desire position and heading. Both neural network control and fuzzy control have the advantage of good robustness. Neural network control performs better in the aspects of control output and decreasing residual.

\subsection{Experiment}

The experiments carried out in this paper are to investigate and verify the effects of NN control algorithm applied to DPS. The experiments were conducted using the model vessel, having a mass of $m=700 \mathrm{~kg}$, length of $L=3.5 \mathrm{~m}$ and breadth of $B=1.0 \mathrm{~m}$. The initial position and heading of experiment are set as $P_{0}=[0 \mathrm{~m}, 0 \mathrm{~m}, 0 \mathrm{rad}]$, and the desired position and heading are $y_{d}=[10 \mathrm{~m}, 10 \mathrm{~m}, 1 \mathrm{rad}]$. The environmental conditions were considered. A wind of approximately $8 \mathrm{~m} / \mathrm{s}$ was simultaneously imposed on the ship. Due to the limitation of thruster system, the maximum force and torque of 3-DOF are limited at $15 N(N \cdot m)$, and the changing extent is less than $1 N(N \cdot m)$ during each cycle. 

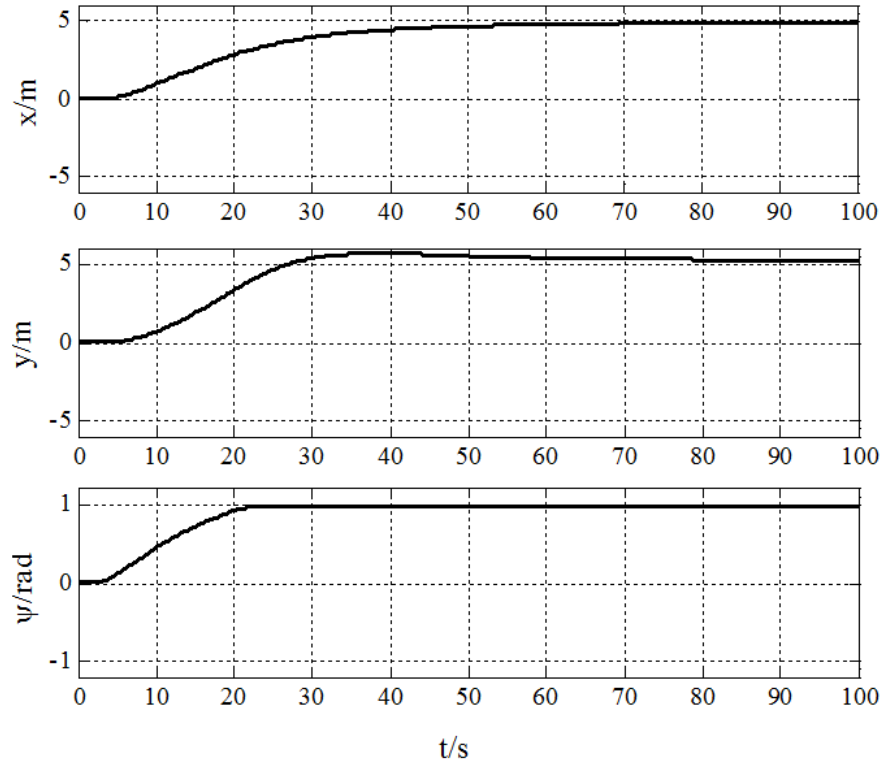

Figure 15: NN control.
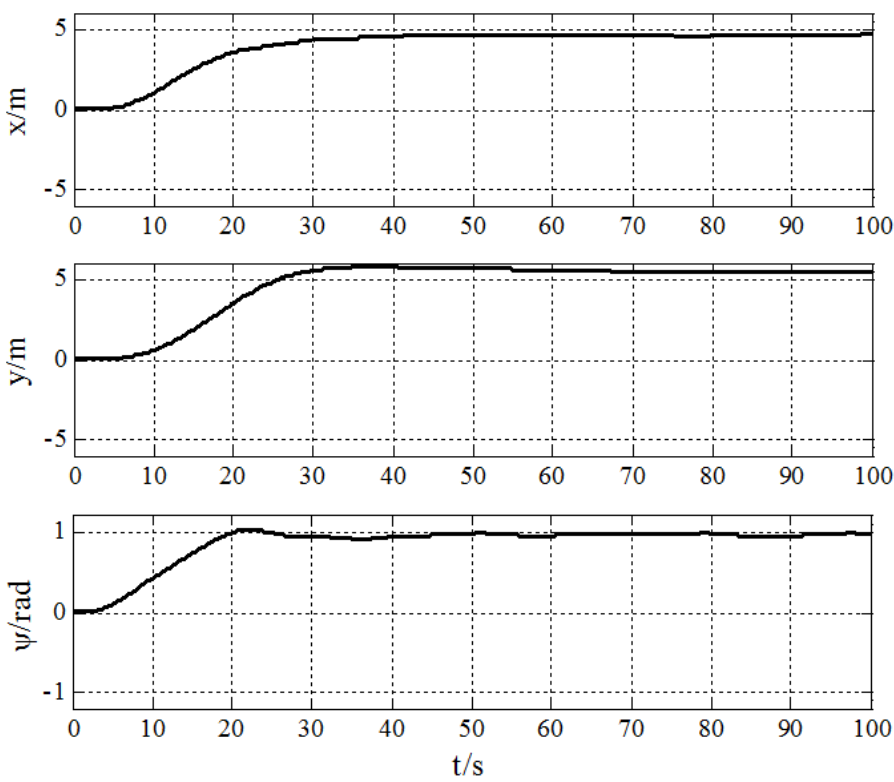

Figure 16: Fuzzy control. 


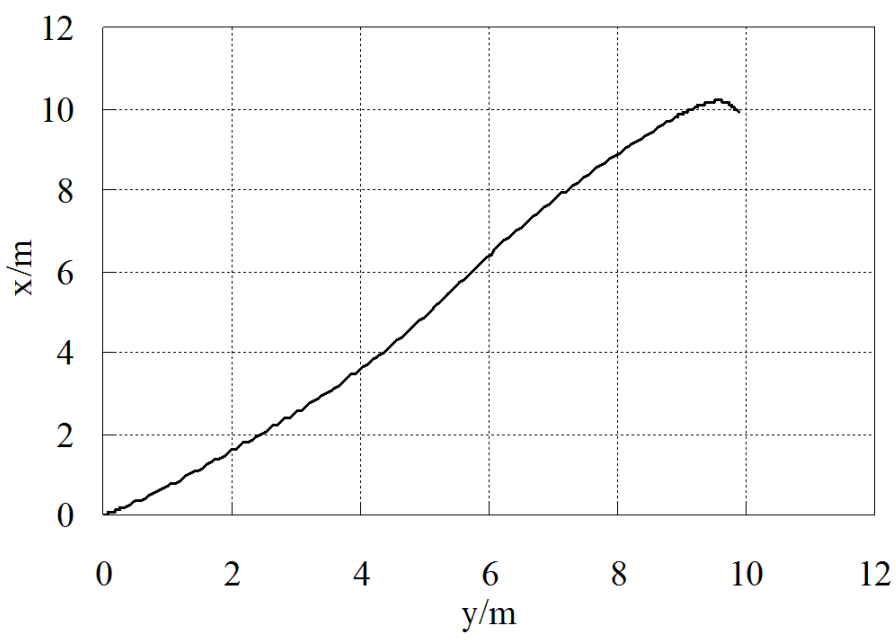

Figure 17: Motion of the ship.
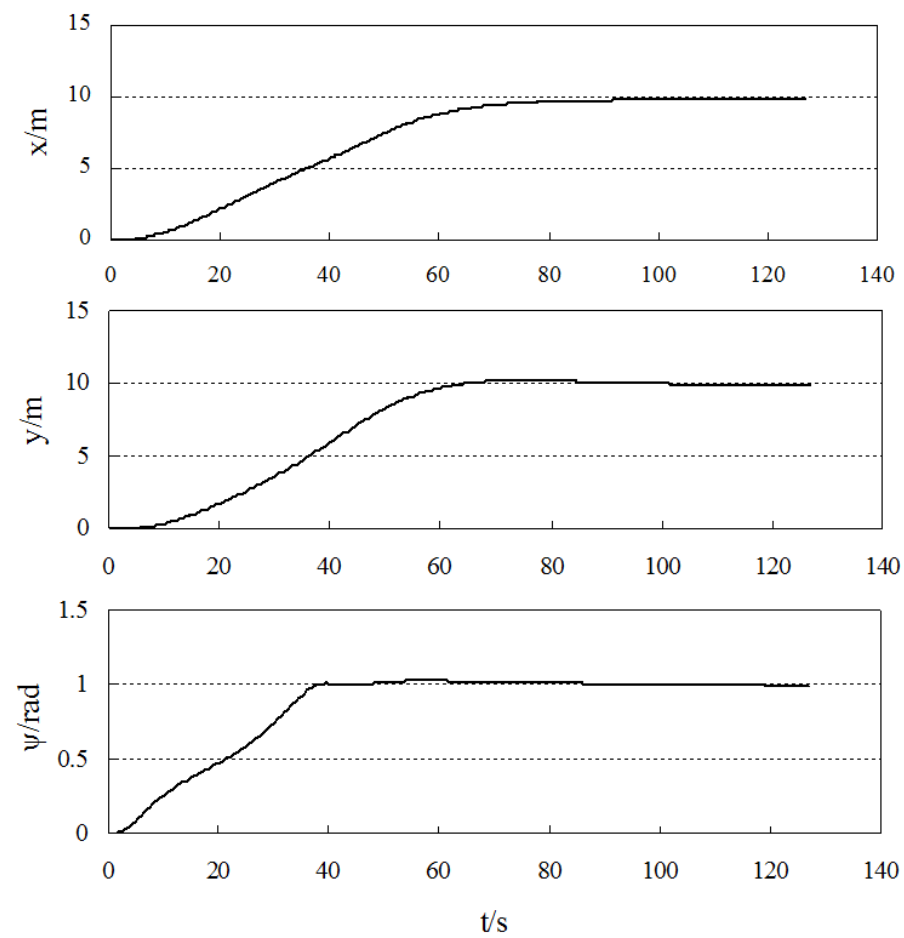

Figure 18: Curves of the $X$ direction, $Y$ direction and heading versus time. 

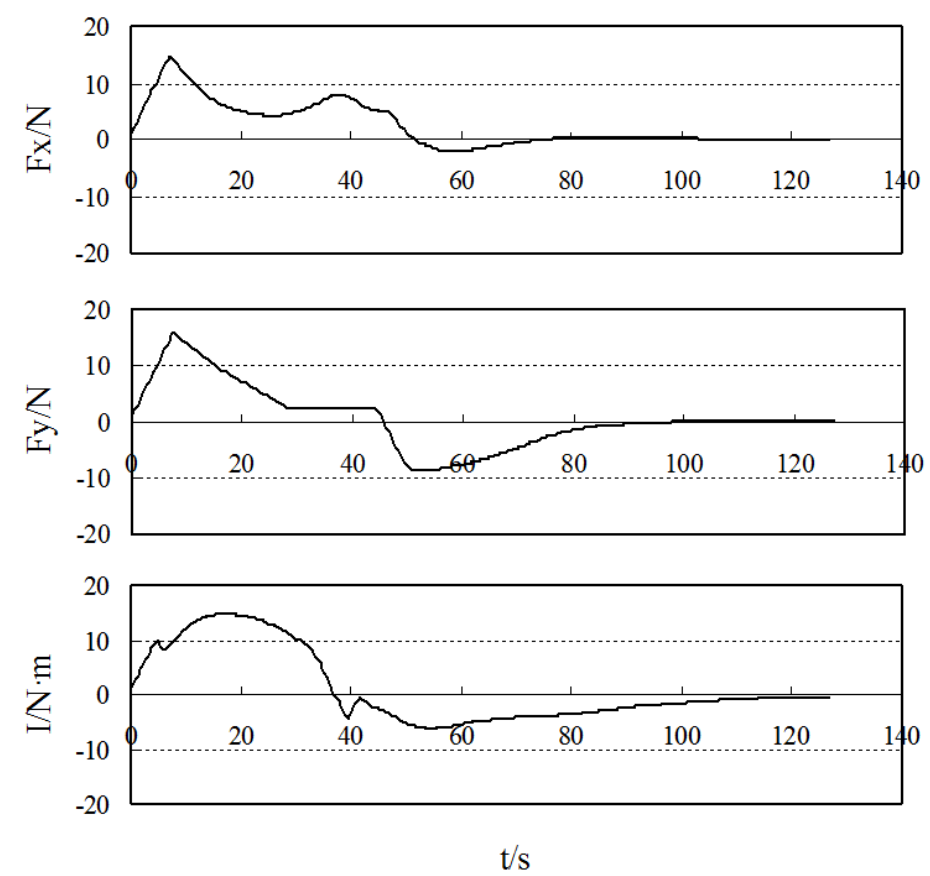

Figure 19: Control force of $X$ direction and $Y$ direction and control torque versus time.

Fig. 17 shows the motion of ship with designed NN controller. The curve of ship motion is an approximate straight line. Fig. 18 shows that the actual ship position $(x, y)$ and the actual heading $\psi$ can approach to the desired position and heading with small overshoot. The corresponding control force and torque are presented in Fig. 19, which is broadly similar to those of previous simulations. It is obviously that the control forces and torque are smooth and reasonable.

\section{Conclusion}

This paper proposed two kinds of intelligent control algorithm for DPS utilizing operator's experience. In the fuzzy control, the experience directly conducts 49 fuzzy rules to participate in process control, while in $\mathrm{NN}$ control, the experience is used to train the neural network. The two control algorithms both maintain the feature of artificial intelligence. Aiming at ship motion, which is a big lag time and complex nonlinear kinematics with changing environmental conditions, fuzzy controller and NN controller are designed. Numerical simulations are provided and indicate that intelligent control is effective for the complex system. By comparison, NN control algorithm performs better in some aspects. The experiments with NN controller for DPS are conducted. The 
results confirmed that when the vessel is exposed to changing environmental conditions, the designed NN control method has high positioning accuracy and good robustness.

\section{References}

[1] A.J. Sorensen: A survey of dynamic positioning control systems. Annual Reviews in Control, 35(1), (2011), 123-136.

[2] Yang Yang, Jialu Du, Guangqiang Li, Wenhua Li and Chen Guo: Dynamic surface control for nonlinear dynamic positioning system of ship. Mechanical Engineering and Technology, 125 (2012), 237-244.

[3] E.A. TAnnuri, H.M. Morishita: Experimental and numerical evaluation of a typical dynamic positioning system. Applied Ocean Research, 28 (2006), 133-146.

[4] Van Phuoc Bui, Sang Won Ji, Kwang Hwan Choi and Young Bok Kim: Nonlinear observer and sliding mode control design for dynamic positioning of a surface vessel. Int. Conf. on Control, Automation and Systems, Jeju Island, (2012), 1900-1904.

[5] N.E. Kahveci, A. Petros and A. Ionnnou: Adaptive steering control for uncertain ship dynamics and stabilityanalysis. Automatica, 49 (2013), 685-697.

[6] P. MARTIN and R. KATEBI: Multivariable PID tuning of dynamic ship positioning control systems. J. of Marine Engineering and Technology,1 (2005), 11-24.

[7] N. AKASAKA: Design of piecewise linear LQ control by multivariable circle criterion for dynamic positioning system. SICE-ICASE Int. Joint Conf., Busan, Korea, (2006), 5672-5677.

[8] Wang Liyun, XiaO Jianmei and Wang Xihuai: Ship dynamic positioning systems based on fuzzy predictive control. Telkomnika, 11 (2013), 6769-6779.

[9] E.W. McGookin, D.J. Murray-Smith, Y. Li and T.I. Fossen: Ship steering control system optimisation using genetic algorithms. Control Engineering Practice, 8 (2000), 429-443.

[10] Zhang Cheng-Du, Wang Xi-Huai and Xiao Jian-MeI: Ship dynamic positioning system based on backstepping control. J. of Theoretical and Applied Information Technology, 51(1), (2013), 129-136.

[11] J. Fotakis, M.J. Grimble and B. Kouvaritakis: A comparison of characteristic locus and optimal designs for dynamic ship positioning systems. IEEE Trans. on Automatic Control, 27(6), (1982), 1143-1157. 
[12] T.I. Fossen: A survey on nonlinear ship control: from theory to practice. Proc. of the 5th IFAC Conf. on Manoeuvring and Control of Marine Craft, Aalborg, Denmark, (2000).

[13] M. Parnichkun and $\mathrm{CH}$. Ngaecharoenkul: Kinematics control of a pneumatic system by hybrid fuzzy PID. Mechatronics, 11 (2001), 1001-1023.

[14] Yong WANG and BAO-ZHU Jia: Fuzzy switching PID controller for ship motion. Navigation of China, 4 (2006), 30-34.

[15] Jia Baozhu, Guang Ren and Zhihong XiU: Fuzzy Switching Controller for Multiple Model. Second Int. Conf. on Fuzzy Systems and Knowledge Discovery, Changsha, China, 1 (2005), 1011- 1014.

[16] Jialu Du, Yang Yang, Dianhui Wang and Chen Guo: A robust adaptive neural networks controller for maritime dynamic positioning system. Neurocomputing, 110 (2013), 128-136.

[17] A. Boulkroune, N. Bounar, M. M'SAAD and M. FarZa: Indirect adaptive fuzzy control scheme based on observer for nonlinear systems: A novel SPR-filter approach. Neurocomputing, 135 (2014), 378-387.

[18] Dong W. Kim, NaK-Hyun Kim and Gwi-Tae Park: Neural network control of humanoid robot. J. of Institute of Control, Robotics and Systems, 16(10), (2010), 963-968.

[19] T.D. Nguyen, A.J. Sfirensen, S.T. Quek: Design of hybrid controller for dynamic positioning from calm to extreme sea conditions. Automatica, 43 (2007), 768-785.

[20] T.I. Fossen: Marine Control Systems: Guidance, Navigation and Control of Ships, Rigs and Underwater Vehicles. Marine Cybernetics, Trondheim, Norway. 2002.

[21] Yao-Qing Ren, XiaO-Gang Duan, Han-Xiong Li and C.L. Philip Chen: Dynamic switching based fuzzy control strategy for a class of distributed parameter system. Journal of Process Control, 24 (2014), 88-97.

[22] S. Chekkal, N.A. Lahacani, D. Aouzellag, K. Ghedamsi: Fuzzy logic control strategy of wind generator based on the dual-stator induction generator. Electrical Power and Energy Systems, 59 (2014), 166-175

[23] T-C. M, J.L Siva Neto and H. Le-HuY: Fuzzy logic based controller for induction motor drives. Proc. of the Canadian Conf. on Electrical and Computer Engineering, 2 (1996), 631-634. 